\title{
Forschung tut not
}

\section{Wissen generieren für die Theorie und für die Praxis Sozialer Arbeit}

\author{
Silke Birgitta Gahleitner und Ingrid Miethe
}

In allen Disziplinen ist Forschung aufgerufen, Grundlagen für die Weiterentwicklung der Theoriebildung sowie für konkrete Praxiskonzepte und Praxisabläufe $z u$ schaffen. Dies gilt insbesondere für die Soziale Arbeit, für deren Entwicklung als eigenständige Fachdisziplin eine grundständige Forschungsorientierung unabdingbar ist.

Ausdruck dieser neuen Orientierung ist die zunehmende Implementierung von Forschungsmethoden in die neuen Bachelor- und Masterstudiengänge und die Schaffung von Forschungs- und Promotionskolloquien. Ein weiterer Beleg für die steigende Bedeutung der Forschung in der Sozialen Arbeit ist der Einsatz für ein eigenes Promotionsrecht an Hochschulen für angewandte Wissenschaften in Deutschland. Auch der im Bolognaprozess formulierte Auftrag praxisnaher For- schen gebunden und haben für diese eine jeweils spezifische Bedeutung. Nur aus (selbst-) reflexiven Praktiken entstehen in einem beständigen Zyklus neue Fragestellungen für Weiterentwicklungen, in der eigenen Berufsidentität wie im kollektiven Professionsverständnis.

Die qualitative rekonstruktive Forschung - mit ihrer Perspektive auf das Subjekt in seiner Eingebundenheit in die Struktur und auf die Struktur in ihrer Wechselwirkung mit Subjekten - kann hier zentrale Wege neuer Verständnishorizonte und Interventionsmöglichkeiten bereitstellen. Von daher ist es kein Zufall, dass Autorinnen und Autoren der Beiträge in diesem Heft ihre eigenen wissenschaftlichen Wurzeln in diesem qualitativen Paradigma verorten und qualitative Methoden nicht nur für die Forschung nutzen, sondern auch für die Handlungspraxis zu modifizieren versuchen. Gleich-

\section{»Praxis schafft Wissen und Wissen schafft Praxis «}

Helmut Pauls, deutscher Psychologe

schung führt zunehmend zu einer stärkeren Forschung an den (Fach-) Hochschulen in Deutschland. Und nicht zuletzt zeigt sich die Wichtigkeit dieses Bereichs auch darin, dass es innerhalb der Deutschen Gesellschaft für Soziale Arbeit e. V. zur Etablierung einer Fachgruppe Forschung kam.

Die Soziale Arbeit ist eine komplexe und angewandte Disziplin mit einem doppelten Mandat, psychosoziale Lebensweisen und Lebenslagen mittels professioneller Methoden zu verstehen und zu verändern. Für sie ist es deshalb von besonderer Bedeutung, Forschung konkret auf Individuen und Gruppen in ihren jeweiligen sozialen Kontexten - Institutionen, Organisationen, Gemeinwesen etc. - auszurichten. Soziale Phänomene sind an (soziale) Erfahrungen von Men- zeitig wird aber auch deutlich, dass der Vielfalt der Sozialen Arbeit kaum mit einzelnen Methoden allein gedient ist, sondern auf die gesamte Palette qualitativer und quantitativer Verfahren zurückgegriffen werden muss. Das Eröffnen alter Dualitäten im Sinne von qualitativ vs. quantitativ wäre eher kontraproduktiv als Erkenntnis fördernd.

Sozialarbeitswissenschaft in Deutschland hat die Aufgabe, die Wechselbeziehung verschiedener Bezugswissenschaften in einer eigenen Wissenschaft der Sozialen Arbeit fruchtbar zu machen. Diese Transdisziplinarität wird inzwischen anstelle eines Mankos immer mehr als Chance aufgefasst. Die in diesem Heft exemplarisch vorgestellten Arbeitsbereiche Jugendhilfe, Wohnungslosigkeit und Interkulturalität weisen anschaulich viele 
dieser interdisziplinären Zugänge, Bezugslinien und Kontextualisierungen auf. Sie geben einen exemplarischen Einblick in die Vielfalt der Forschungs- und Praxisthemen Sozialer Arbeit.

Forschung in der Sozialen Arbeit hat viele Aufgaben. Im Prozess der reflexiven Modernisierung und Verwissenschaftlichung stellt sie neue Möglichkeiten zur Verfügung, sich ständig verändernde und hoch komplexe Prozesse zu erfassen und situationsadäquate Handlungsmöglichkeiten zu entfalten. Forschung soll auch Wissen über und Verständnis für psychosoziale Probleme generieren und dazu beitragen, Strategien zu entwickeln und wirksamer zu handeln. Sie sollte situationsspezifisch und zielgruppengenau in der Lebenswelt ansetzen und empirisch belegen, was sich in der Praxis bewährt.
Vor allem anderen jedoch ist Forschung in der Sozialen Arbeit Teil professioneller Praxis vor Ort und wird von Sozialarbeiterinnen und Sozialarbeitern benötigt wie unterstützt. Sie ist ein selbstverständlicher Teil tagtäglicher Arbeitsrealität, was eine sozialarbeiterische Grundlagenforschung mit einschließt, die erforderlich ist, um die Fachwissenschaft als Ganzes weiter zu entwickeln.

\section{Buchreihe informiert über »Rekonstruktive Forschung in der Sozialen Arbeit«}

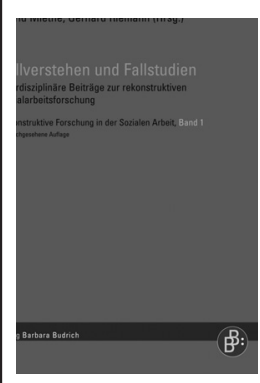

In der Diskussion um eine Sozialarbeitswissenschaft als eigenständige Disziplin geht es derzeit primär darum, den Gegenstand dieser Disziplin und ihr Verhältnis $\mathrm{zu}$ angrenzenden Bezugsdisziplinen wie Soziologie, Sozialpädagogik oder Psychologie zu bestimmen. Wird darüber hinaus die Frage der Vermittlung zwischen sozialwissenschaftlicher Theorie und sozialarbeiterischer Praxis erörtert, welche für die Sozialarbeitswissenschaft ebenfalls zentral ist, so ist festzustellen, das der Forschung ein hoher Stellenwert beigemessen wird.

Hier setzt die von Wolfram Fischer, Cornelia Giebler, Martina Goblirsch, Ingrid Miethe und Gerhard Riemann im Verlag Barbara Budrich, Opladen \& Farmington Hills, herausgegebene Buchreihe »Rekonstruktive Forschung in der Sozialen Arbeit « an. Dass auf die rekonstruktive Sozialforschung zurückgegriffen wird, liegt nahe, denn deren Grundannahme der Konstruiertheit sozialer Wirklichkeit ebenso wie ihre methodischen Prinzipien, die den Forschungssubjekten Gelegenheit geben, die eigenen Relevanzsysteme kommunikativ zu entfalten, sind in besonderer Weise geeignet, an eine auf das Verstehen fremder Lebenswelten ausgerichtete Praxis Sozialer Arbeit anzuschließen.

Die Buchreihe setzt sich bislang aus zwei Sammelbänden und vier Monografien zusammen. Die Sammelbände sind jeweils im Nachgang zu Tagungen des Netzwerks »Rekonstruktive Sozialarbeitsforschung und Biografie « entstanden. In ihnen finden sich zum einen Beiträge zu aktuellen Forschungsarbeiten, welche sich auf unterschiedliche Felder sozialer Arbeit beziehen und die Klientel Sozialer Arbeit ebenso in den Blick nehmen wie die professionell Tätigen. Ferner setzen sich mehrere Autorinnen und Autoren mit der Nutzung von Methoden rekonstruktiver Sozialforschung für die Selbstreflexion im Kontext des Studiums der Sozialen Arbeit wie auch für die Selbstbeforschung der Praxis auseinander. Noch über diese Intentionen hinausgehend wird danach gefragt, wie die Methoden modifiziert werden können, um unmittelbar in die sozialarbeiterische Praxis Eingang zu finden. Und schließlich geht es in verschiedenen Beiträgen um die Frage, welche verbindenden Elemente einer rekonstruktiven Sozialarbeitsforschung sich herauskristallisieren, wenn man die Erfahrungen aus Forschung, Praxis und Lehre zusammenführt.

In den vier Monografien (Loch 2006, Müller 2006, Lehmann 2008, Sauer 2008) werden mittels unterschiedlicher Metho- den rekonstruktiver Sozialforschung Fragestellungen behandelt, welche aus der sozialarbeiterischen Praxis heraus entwickelt wurden oder für die Beratungsausbildung in der Sozialen Arbeit relevant sind. Es geht beispielsweise um die $\mathrm{Zu}-$ sammenarbeit von Pflege- und Herkunftsfamilien in dauerhaften Pflegeverhältnissen (vgl. Sauer 2008) oder Migrantinnen im Frauenhaus (vgl. Lehmann 2008). Positiv fällt auf, dass auf der Ebene der Forschung die Bestimmung des Verhältnisses der Sozialarbeitswissenschaft zu ihren Bezugsdisziplinen praktisch gelöst wird: Hier stehen konkrete Praxisprobleme der Sozialen Arbeit im Mittelpunkt und Erkenntnisse verschiedener Disziplinen werden, wie es für rekonstruktive Sozialforschung charakteristisch ist, ausgehend von den Fallrekonstruktionen und Typologien hinzugezogen. Die Buchreihe insgesamt zeichnet sich dadurch aus, dass mit ihr ein Forum geschaffen wurde, in dem Forschende, Lehrende, Praktikerinnen und Praktiker ebenso wie Studierende in den Dialog treten, um im Raum zwischen Theorie und Praxis gemeinsam Neues zu entwickeln. Sie leistet damit einen wichtigen Beitrag zur Professionalisierung sozialer Arbeit wie auch zur Forschung in der Sozialarbeitswissenschaft.

Sonja Kubisch

Dr. Sonja Kubisch ist wissenschaftliche Referentin im Bundesnetzwerk Bürgerschaftliches Engagement. E-Mail sonja.kubisch@b-b-e-de

Band 1: Cornelia Giebeler, Wolfram Fischer, Martina Goblirsch, Ingrid Miethe, Gerhard Riemann (Hrsg.) (2007): Fallverstehen und Fallstudien. Interdisziplinäre Beiträge zur rekonstruktiven Sozialarbeitsforschung. ISBN 978-3-86649-207-3.

Band 2: Ulrike Loch (2006): Sexualisierte Gewalt in Kriegs- und Nachkriegskindheiten. Lebens- und familiengeschichtliche Verläufe. ISBN 978-3-86649-070-3.

Band 3: Jutta Müller (2006): Coaching, Biografie und Interaktion. Eine qualitative Studie zum Coach in Ausbildung. ISBN 978-386649-063-5.

Band 4: Ingrid Miethe, Wolfram Fischer, Cornelia Giebeler, Martina Goblirsch, Gerhard Riemann (Hg.) (2007): Rekonstruktion und Intervention. Interdisziplinäre Beiträge zur rekonstruktiven Sozialarbeitsforschung. ISBN 978-3-86649-082-6.

Band 5: Stefanie Sauer (2008): Die Zusammenarbeit von Pflegefamilie und Herkunftsfamilie in dauerhaften Pflegeverhältnissen. Widersprüche und Bewältigungsstrategien doppelter Elternschaft. ISBN 978-3-86649-124-3.

Band 6: Nadja Lehmann (2008): Migrantinnen im Frauenhaus. Biographische Perspektiven auf Gewalterfahrungen. ISBN 978-3-86649159-5. 